\title{
Arterial stiffness is a predictor for acute kidney injury following coronary artery bypass graft surgery
}

Sharlene A. Greenwood ${ }^{1,2,3^{*}}$ (D), Emmanuel Mangahis ${ }^{1,2}$, Ellen M. Castle ${ }^{1,2,3}$, Joe Wang ${ }^{4}$, Jackie Campbell ${ }^{5}$, Ranjit Deshpande ${ }^{6}$ and Satish Jayawardene $e^{2,3}$

\begin{abstract}
Background: Cardiac surgery-associated acute kidney injury (CSA-AKI) is a serious postoperative complication of cardiac surgery, an episode of which impacts on patient morbidity and mortality. Pulse wave velocity (PWV; a noninvasive measurement tool to assess arterial stiffness) has been shown to predict kidney disease progression, and cardiovascular and all-cause mortality in patients with chronic kidney disease. We hypothesised that PWV would also predict acute kidney injury in subjects who have undergone non-valve repair elective coronary artery bypass graft (CABG) surgery .
\end{abstract}

Methods: This was a prospective, observational, exploratory study. PWW was determined with a Vicorder device, together with standard clinical and biochemical parameters. AKI staging was defined according to the Kidney Disease Improving Global Outcomes (KDIGO) Clinical Practice Guidelines.

Results: 137 patients were included in the study. 85\% were male, and mean age was 66.3 years (SD $=9.7$ years). There were 40 episodes (29\%) of CSA-AKI. Each 1 unit increase in PWV score was associated with a 1.5 fold greater odds of a CSA-AKI event ( $p=0.006$ (odds ratio $=1.5$; confidence interval:1.13-2.10). A 1 unit increase in estimated glomerular filtration rate resulted in an estimated $85 \%$ decrease in the odds of developing AKI, each year, men have an odds reduction of 15\% of developing AKI compared with females and each 1 year increase in age lowered the odds of developing AKI by $87 \%$.

Conclusions: This pilot exploratory study revealed that PWV, assessed prior to non-valve repair elective CABG surgery, independently predicts CSA-AKI events. PWV is a simple, non-invasive technique that could potentially be used to risk stratify for CSA- AKI following elective cardiac surgery.

Trial registration: ClinTrial.Gov NCT02364427. Registered 18 February 2015.

Keywords: Arterial stiffness, Pulse wave velocity, Acute kidney injury, Coronary artery bypass graft surgery

\section{Background}

Acute Kidney Injury (AKI) affects around 20\% of all hospitalised patients [1] and has both major long-term health and socioeconomic implications [2, 3]. Longitudinal studies have demonstrated that an episode of AKI can significantly increase both the risk of development of chronic kidney disease and early mortality [4]. Published data that considers non-valve repair coronary

\footnotetext{
* Correspondence: sharlene.greenwood@nhs.net

${ }^{1}$ Department of Therapies, King's College Hospital, London, UK

2Department of Renal Medicine, King's College Hospital, London, UK

Full list of author information is available at the end of the article
}

artery bypass graft (CABG) patients report an incidence rate of AKI to be between 2 and 12\% [5, 6]. Our research team performed a retrospective analysis of incidence of AKI in patients undergoing elective, isolated CABG surgery at our hospital from January to December 2012. Of a total of 219 patients, 42 patients were classified as having post-operative AKI according to serum AKI criteria (unpublished data). This represents an incidence of $19.6 \%$.

Currently there is no specific therapy for AKI and the management of patients is completely supportive. Furthermore, in the absence of more sensitive biomarkers,

(c) The Author(s). 2019 Open Access This article is distributed under the terms of the Creative Commons Attribution 4.0 International License (http://creativecommons.org/licenses/by/4.0/), which permits unrestricted use, distribution, and 
it would appear that even a non-severe episode of AKI already confers a worse prognosis with regards to the risk of development of CKD and long term survival in patients. Thus perhaps focus should be on prevention, and early detection of risk, of AKI as opposed to cure. This is even more pertinent when one considers that the National Confidential Enquiry into Patient Outcomes and Death (NCEPOD) report in 2009 [1] identified 30\% of cases of AKI as being 'preventable' and a deficiency of care in $50 \%$ of cases. Thus 'preventable' cases of AKI could potentially save $£ 130-186$ million per annum [3].

Although factors such as diabetes, older age and low eGFR are all associated with increased risk of AKI, there is no standardized scoring method for risk [7]. In addition, it is not fully clear why some patients suffer AKI while others, with similar co-morbidities, do not. Arterial stiffness (AS) can be assessed non-invasively by measurement of pulse wave velocity (PWV) [8]. Kidney function is highly reliant on renal perfusion and good vascular health. This relationship is emphasised by studies that show renal functional decline is associated with an increase in risk of adverse cardiovascular events and death [9]. This has led to a focus on 'non-traditional' risk factors in the renal population such as AS and left ventricular hypertrophy (LVH). Increased AS has been shown to be be an independent risk factor for cardiovascular events in ESRF and in renal transplant recipients $[10,11]$.

The aim of this exploratory study was to investigate whether arterial stiffness, as defined by measuring Pulse Wave Velocity, a simple non-invasive measurement score, could be identified as an independent risk factor for development of post-coronary artery bypass graft acute kidney injury.

\section{Methods}

\section{Participants}

Participants were approached during routine surgical pre-assessment clinic at King's College Hospital (KCH) in London. They were included if they were aged $>18$ years, due to have CABG surgery alone with no concomitant valve surgery, and were able to give written consent. Patients were excluded if they had aortic grafts or renal stents, had renal function reflecting CKD stage 4 or 5 , were receiving dialysis treatment, or had a significant psychiatric illness (including anxiety, mood and untreated eating disorders).

\section{Study procedure}

Patients were assessed in a quiet treatment room in the pre-assessment clinic in the early afternoon. Data collection included blood and urine samples, patient demographics and anthropometrics (height, weight, and waist circumference), followed by blood pressure (BP), heart rate (HR), and PWV. All outcome assessments were performed at the same study visit.

\section{Primary outcome}

Pulse Wave Velocity (PWV), a measurement of arterial stiffness, was assessed at the systemic region (carotid-femoral PWV), which is the gold standard method [12], using the Vicorder system (Skidmore Industries, UK). Conditions for assessment, as stated expert consensus [12], were adhered to for all measurements. The measurement protocol by Hickson et al. [13] was used, mathematically removing the additional femoral segment from the Vicorder standard protocol, to correct for any inherent bias at high arterial PWV. The average of 3 measurements (of 20 consecutive signals) was recorded at each time point.

\section{Acute kidney injury}

The development of AKI was determined according to the Kidney Disease Improving Global Outcomes (KDIGO) Clinical Practice Guideline for Acute Kidney Injury criteria [14]. In brief, the guideline covers definition and staging of AKI based on the Risk, Injury, Failure; Loss, End-Stage Renal Disease (RIFLE) and Acute Kidney Injury Network (AKIN) criteria and studies on risk relationships.

\section{Blood tests}

Venous blood was collected at the study visit. Serum creatinine (traceable to a calibrator tested with a reference method (IDMS - isotope dilution mass spectrometry) was analysed in the biochemistry department at King's College Hospital.

\section{Resting BP and HR}

After sitting quietly for $5 \mathrm{~min}$, resting $\mathrm{BP}$ and $\mathrm{HR}$ were recorded in triplicate, with a 1-min interval between measurements, using an automated sphygmomanometer (Tango; SunTech Medical, Oxfordshire, UK). The average of 3 readings was recorded.

\section{Statistical analyses}

Parameters are expressed as means \pm SD (for normally distributed data) and median and interquartile range for non-parametric data. Differences between groups were assessed by the independent samples median test, $\mathrm{t}$-test, or chi-squared test as appropriate to the data type. Correlations were assessed by the Pearsons correlation coefficient. Linear and logistic regression analyses were used to generate regression beta coefficients (B) and odds ratios for a range of baseline variables (age at assessment, gender, diabetes, eGFR) to determine factors that independently predicted the risk of a post-operative AKI event. 


\section{Results}

A total of 137 participants were recruited to this study over a 24 month period; demographic data and baseline measurements are shown in Table 1. Participants were predominantly male $(85 \%, n=116)$ with a mean age of 66.3 years ( $\mathrm{SD}=9.7$ years), and the majority of the participants were of white ethnicity $(82 \%, n=101)$. None of the participants had a history of pre-operative renal disease or were in an unstable state pre-operatively. Forty patients (29\%) developed AKI according to the KDIGO criteria [14]. Table 1 shows a comparison of the variables at the study assessment between those patients who suffered a post-operative AKI event (AKI) and those patients who did not (No AKI). Statistical significance is defined as $\mathrm{p}_{\mathrm{HO}}<0.05$ throughout. Figure 1 displays Pulse Wave Velocity values and incidence of cardiac surgery associated Acute Kidney Injury.

Patients who suffered an AKI event had lower values of eGFR at baseline $(p<0.001)$, a higher probability of having diabetes, $(p=0.007)$, and a greater Pulse Wave Velocity $(p=0.049)$ compared to those in the no AKI group. A higher percentage of women had AKI than men.

It is recognised that multiple tests will result in inflation of overall Type I error but these results are being used as preliminary indicators of possible predictors in the modelling process. All variables were initially included in the models.

Logistic regression utilising a backwards step-regression model as an exploratory analysis, followed by confirmatory hierarchical modelling using forced entry was used to determine which factors independently predicted a post-operative AKI event after checking for the validity of the analytical assumptions. Factors used in the hierarchical models were added in the following order, determined by the strength of the association with AKI in univariate tests (strongest association first):

- eGFR

- Diabetes
- Pulse Wave Velocity

- Gender

- Age

- Systolic blood pressure

- Heart rate

- Ejection fraction category

It is recommended that the number of predictor variables for logistic regression modelling is limited to one predictor for every 10 events, where an event is defined as the outcome of interest (in this case, an occurrence of AKI) [15]. This has been modified more recently to (a cautious) 5-9 events per variable [16]. There were 20 reported cases of AKI (the outcome measure) in the data and therefore a maximum of 4 predictors are recommended for this analysis, as follows;-.

- eGFR

- pulse wave velocity

- age

- gender

This is in agreement with the exploratory backwards stepwise model. A final, forced entry model was constructed with these predictor variables. This final model has sufficient numbers of events for stability with four predictors. eGFR, age, gender, PWV and participants receiving diabetic management were all statistically significant predictors of post-operative AKI and. as shown in Table 2.

\section{Discussion}

This is the first study to investigate the relationship between PWV and development of AKI post elective CABG surgery; there is, however, previously published data that supports an association between increased levels of AS and reduced kidney function [9]. The results of this current exploratory study demonstrate that higher levels of kidney function (as measured by eGFR), being male, and being younger were associated with a

Table 1 Summary of descriptive statistics and univariate tests for association with acute kidney injury

\begin{tabular}{llll}
\hline Variable & No AKI & AKI & $p$ \\
\hline Age (years) & $67.0(61.0-73.7)^{\mathrm{b}}$ & $71.5(56.7-74.7)^{\mathrm{b}}$ & $0.405^{*}$ \\
Systolic blood pressure (mm Hg) & $133.8(17.3)^{\mathrm{a}}$ & $136.0(10.9)^{\mathrm{a}}$ & $0.578^{\mathrm{s}}$ \\
Heart rate (bps) & $65.0(56.0-72.0)^{\mathrm{b}}$ & $66.5(59.0-76.2)^{\mathrm{b}}$ & $0.645^{*}$ \\
Pulse wave velocity $\left(\mathrm{ms}^{-1}\right)$ & $8.3(7.3-9.9)^{\mathrm{b}}$ & $9.3(8.4-10.7)^{\mathrm{b}}$ & $0.049^{*}$ \\
eGFR $\left(\mathrm{ml}^{-1} \cdot \mathrm{min}^{-1} .1 .73 \mathrm{~m}^{-2}\right)$ & $78.5(68.2-90.0)^{\mathrm{b}}$ & $53.0(42-65.2)^{\mathrm{b}}$ & $<0.001^{*}$ \\
Diabetes (\%yes) & $21.4 \%$ & $50.0 \%$ & $0.007^{\#}$ \\
Ejection fraction category (\%good) & $69.2 \%$ & $65.0 \%$ & $0.912^{\#}$ \\
Gender (\%male) & $87.2 \%$ & $70.0 \%$ & $0.049^{\#}$ \\
\hline
\end{tabular}

(Descriptive statistics: ${ }^{\text {a }}$ denotes mean $(\mathrm{sd}){ }^{\mathrm{b}}$ denotes median (IQR). Statistical tests: ${ }^{*}$ denotes independent samples median test; $\$$ denotes independent groups ttest; \# denotes chi-squared test) 


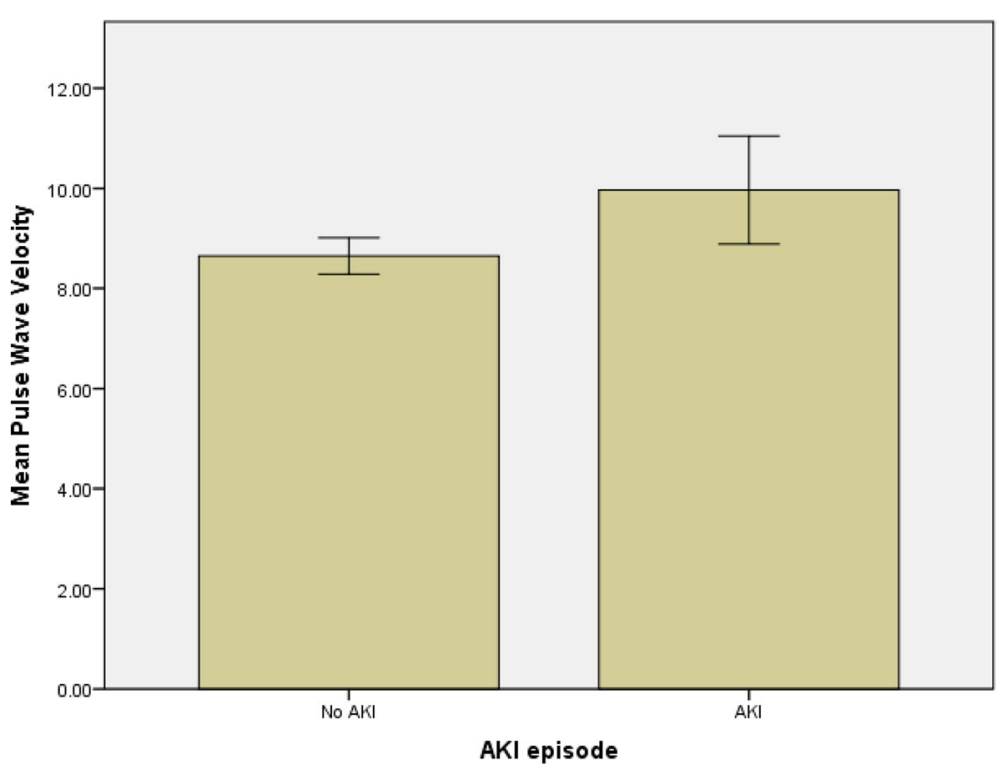

Fig. 1 Mean Pulse Wave Velocity values (95\% Cl) and incidence of cardiac surgery associated Acute Kidney Injury. Mean pulse wave velocity (m/ sec); AKI = Acute Kidney Injury

lower risk of participants developing post-operative AKI. These results are consistent with previous studies [7] demonstrating preoperative renal impairment (eGFR < $60 \mathrm{ml} / \mathrm{min}$, creatinine $>2.1 \mathrm{mg} / \mathrm{dl}$ ) and female sex as independent risk factors for Cardiac Surgery associated AKI (CSA-AKI). Our study also revealed a significant association between pre-operative arterial stiffness (as measured by PWV) and the risk of developing AKI after elective CABG surgery. There was an increased odds ratio of developing AKI of 1.5 with every unit increase in PWV. Earlier work by Kidher et al. [17] reported a non-significant weak correlation between PWV and post aortic valve replacement surgery-AKI in patients with normal to mild levels of pre-operative renal impairment. This current study, which benefited from a larger sample of participants with normal to mild pre-operative renal impairment, appears to contradict this earlier work that didn't show a significant association between PWV and CSA-associated AKI although we do recognise that the cardiac surgery between the two studies is different.

The mechanism of injury in CKD from increased AS is thought to be related to barotrauma inflicted on glomeruli in a "stiff" vascular system [9]. Preventive strategies for CSA-AKI are limited and the evidence for most interventional therapies is not substantive. The use of a simple non-invasive AS measurement technique, such as PWV, was shown to offer a quick and reliable way to predict those patients at risk of CSA-AKI in our exploratory study. As arterial stiffness is potentially modifiable with therapeutic strategies, reducing AS in potential candidates for cardiac surgery at high risk for AKI, may reduce the risk of CSA-AKI. By identifying those patients at risk of AKI, there can also be increased awareness and vigilance pre- and post-cardiac surgery.

Several limitations need to be acknowledged. Firstly, our study consists of a predominantly caucasian population undergoing elective non-valve repair CABG surgery so there is a need be cautious when extrapolating these results to other patient populations. Secondly, the small number of post-operative AKI episodes meant that we had to restrict the number of dependent variables in the multivariate model. Measurement of PWV as a predictor of CSA-AKI in a larger patient population would be of interest.

Table 2 Final model parameters

\begin{tabular}{|c|c|c|c|c|c|c|c|}
\hline & \multirow[t]{2}{*}{ B } & \multirow[t]{2}{*}{ S.E. } & \multirow[t]{2}{*}{ Wald } & \multirow[t]{2}{*}{$p$} & \multirow[t]{2}{*}{$\operatorname{Exp}(B)$} & \multicolumn{2}{|c|}{ 95\% C.I.for EXP(B) } \\
\hline & & & & & & Lower & Upper \\
\hline eGFR & -.157 & .035 & 20.211 & $<.001$ & .855 & .798 & .915 \\
\hline PWV & .431 & .158 & 7.453 & .006 & 1.538 & 1.129 & 2.096 \\
\hline Age & -.140 & .052 & 7.271 & .007 & .870 & .786 & .963 \\
\hline Gender & -1.912 & .896 & 4.555 & .033 & .148 & .026 & .855 \\
\hline Constant & 15.396 & 4.815 & 10.226 & .001 & $4,856,062.071$ & & \\
\hline
\end{tabular}




\section{Conclusion}

The results from this exploratory study indicate that PWV, assessed prior to elective non-valve repair CABG surgery, independently predicts post-operative AKI events. PWV is a simple, non-invasive technique that could potentially be used to help in stratifying the risk of post-operative AKI following cardiac surgery. Importantly, unlike other risk factors such as eGFR, there is potential to modify AS pre-cardiac surgery and reduce the risk of CSA-AKI. Further research to evaluate potential therapeutic strategies to reduce AS is warrented.

\begin{abstract}
Abbreviations
AKI: Acute kidney injury; AKIN: Acute Kidney Injury Network; AS: Arterial stiffness; BP: Blood pressure; CABG: Coronary artery bypass graft; CKD: Chronic kidney disease; CSA-AKI: Cardiac surgery-associated acute kidney injury; eGFR: Estimated glomerular filtration rate; ESRF: End stage renal failure; HR: Heart rate; IDMS: Isotope dilution mass spectrometry; KCH: King's College Hospital; KDIGO: Kidney Disease Improving Global Outcomes; LVH: Left ventricular hypertrophy; NCEPOD: National Confidential Enquiry into Patient Outcomes and Death; NIHR/HEE: National Institute for Health Research/Health Education England; PWV: Pulse Wave Velocity; RIFLE: Risk, Injury, Failure; Loss, End-Stage Renal Disease
\end{abstract}

\section{Acknowledgements}

The study team would like to acknowledge the help of lain Macdougall in the preparation of the manuscript.

\section{Funding}

Sharlene Greenwood is funded by a National Institute for Health Research/ Health Education England (NIHR/HEE) Clinical Lectureship. The views expressed are those of the author(s) and not necessarily those of the NHS, the NIHR or the Department of Health. The funders did not have any role in study design; collection, analysis, and interpretation of data; writing the report; and the decision to submit the report for publication.

\section{Availability of data and materials}

The datasets used and/or analysed during the current study are available from the corresponding author on reasonable request.

\section{Authors' contributions}

Research idea and study design: SG, EC, JM, HL, DG, EM, IW, HM, ICM; data acquisition: SG, EC, JM, HL, DG, EM, IW, OC, KS, HM, ICM; data analysis/ interpretation: SG, EC, JM, HL, DG, EM, IW, OC, KS, HM, ICM; statistical analysis: SG, EC, HM, ICM. Each author contributed important intellectual content during manuscript drafting or revision and accepts accountability for the overall work by ensuring that questions pertaining to the accuracy or integrity of any portion of the work are appropriately investigated and resolved. SG and HM take responsibility that this study has been reported honestly, accurately, and transparently; that no important aspects of the study have been omitted; and that any discrepancies from the study as planned and registered have been explained. All authors read and approved the final manuscript.

\section{Ethics approval and consent to participate}

The study was approved by Wales REC 6, and was registered with ClinTrial.Gov: NCT02364427. Eligible patients were provided with a patient information sheet, and were given an opportunity to ask questions. Following written informed consent, assessments were conducted on the same day.

\section{Consent for publication}

Not applicable

\section{Competing interests}

The authors declare that they have no competing interests.

\section{Publisher's Note}

Springer Nature remains neutral with regard to jurisdictional claims in published maps and institutional affiliations.

\section{Author details}

${ }^{1}$ Department of Therapies, King's College Hospital, London, UK. ${ }^{2}$ Department of Renal Medicine, King's College Hospital, London, UK. ${ }^{3}$ Renal Sciences, Department of Transplantation, Immunology and Mucosal Biology, King's College London, London, UK. ${ }^{4}$ Department of Renal Medicine, Epsom \& St Helier Hospital, London, UK. ${ }^{5}$ Faculty of Health and Social Care, University of Northampton, Northampton, UK. ${ }^{6}$ Department of Cardiovascular Sciences, King's College Hospital, London, UK.

Received: 23 January 2019 Accepted: 20 February 2019

Published online: 07 March 2019

\section{References}

1. National Confidential Enquiry into Patient Outcomes and Death (NCEPOD) Report: Adding Insult to Injury. 2009.

2. Hsu CY, Chertow GM, McCulloch CE, Fan D, Ordonez JD, Go AS. Nonrecovery of kidney function and death after acute on chronic renal failure. Clin J Am Soc Nephrol. 2009;4(5):891-8. https://doi.org/10.2215/CJN. 05571008 Epub 2009 Apr 30.

3. Kerr M. The economic impact of acute kidney disease [NHS Kidney Care report.]; 2011.

4. Lassnigg A, Schmidlin D, Mouhieddine M, Bachmann LM, Druml W, Bauer P, et al. Minimal changes of serum creatinine predict prognosis in patients after cardiothoracic surgery: a prospective cohort study. J Am Soc Nephrol. 2004;15(6):1597-605.

5. Olsson D, Sartipy U, Braunschweig F, Holzmann MJ. Acute kidney injury following coronary artery bypass surgery and long-term risk of heart failure. Circ Heart Fail. 2013;6(1):83-90. https://doi.org/10.1161/CIRCHEARTFAILURE. 112.971705 Epub 2012 Dec 10.

6. Layton JB, Kshirsagar AV, Simpson RJ Jr, Pate V, Jonsson Funk M, Sturmer T, et al. Effect of statin use on acute kidney injury risk following coronary artery bypass grafting. Am J Cardiol. 2013;111(6):823-8. https://doi.org/10. 1016/j.amjcard.2012.11.047 Epub Dec 28.

7. Vives M, Wijeysundera D, Marczin N, Monedero P, Rao V. Cardiac surgeryassociated acute kidney injury. Interact Cardiovasc Thorac Surg. 2014;18(5): 637-45. https://doi.org/10.1093/icvts/ivu014 Epub 2014 Feb 16.

8. Bramwell JC, Hill AV. The velocity of transmission of the pulse wave and elasticity of arteries. Lancet. 1922;1:891-2.

9. Wang MC, Tsai WC, Chen JY, Huang JJ. Stepwise increase in arterial stiffness corresponding with the stages of chronic kidney disease. Am J Kidney Dis. 2005;45(3):494-501. https://doi.org/10.1053/j.ajkd.2004.11.011.

10. Al-Aly Z, Zeringue A, Fu J, Rauchman MI, McDonald JR, El-Achkar TM, et al. Rate of kidney function decline associates with mortality. J Am Soc Nephrol. 2010:21(11):1961-9 doi: 10.681/ASN.2009121210. Epub 2010 Oct 14.

11. Bahous SA, Stephan A, Blacher J, Safar M. Cardiovascular and renal outcome in recipients of kidney grafts from living donors: role of aortic stiffness. Nephrol Dial Transplant. 2012;27(5):2095-100. https://doi.org/10. 1093/ndt/gfr578 Epub 2011 Oct 6.

12. Laurent S, Cockcroft J, Van Bortel L, Boutouyrie P, Giannattasio C, Hayoz D, et al. Expert consensus document on arterial stiffness: methodological issues and clinical applications. Eur Heart J. 2006;27(21):2588-605.

13. Hickson SS, Butlin M, Broad J, Avolio AP, Wilkinson IB, McEniery CM. Validity and repeatability of the Vicorder apparatus: a comparison with the SphygmoCor device. Hypertens Res. 2009;32(12):1079-85.

14. (KDIGO) KDIGO. Kidney Disease Improving Global Outcomes Clinical Practice Guideline for Acute Kidney Injury. 2011.

15. Peduzzi P, Concato J, Kemper E, Holford TR, Feinstein AR. A simulation study of the number of events per variable in logistic regression analysis. J Clin Epidemiol. 1996;49(12):1373-9.

16. Vittinghoff $E$, McCulloch CE. Relaxing the rule of ten events per variable in logistic and cox regression. Am J Epidemiol. 2007;165(6):710-8. https://doi. org/10.1093/aje/kwk052 Epub 2006 Dec 20.

17. Kidher E, Harling L, Ashrafian H, Naase H, Chukwuemeka A, Anderson J, et al. Pulse wave velocity and neutrophil gelatinase-associated lipocalin as predictors of acute kidney injury following aortic valve replacement. J Cardiothorac Surg. 2014:9:89. https://doi.org/10.1186/749-8090-9-89. 